\title{
Synaptogenesis in cell cultures of neurones and myotubes from chickens with muscular dystrophy
}

\author{
JOHN H. PEACOCK ${ }^{1}$ AND PHILLIP G. NELSON \\ From the Behavioral Biology Branch, National Institute of Child Health and Human \\ Development, National Institutes of Health, Bethesda, Maryland 20014, U.S.A.
}

SUMMARY Intracellular microelectrode recordings from chick dystrophic myotubes in cell culture reveal a capability for innervation by neurones from either dystrophic or normal embryos. Neither neuronal class differentially affects the incidence of synapse formation at neuromuscular junctions (about $75 \%$ ) or at neural junctions (about $85 \%$ ), the PSP frequency (about $10 / \mathrm{sec}$ ), the maximum quantal content at neuromuscular junctions (over 70), or the resting membrane potentials of either myotubes (about $-53 \mathrm{mV}$ ) or neurones (about $-43 \mathrm{mV}$ ). In each culture condition about $20 \%$ of nerve-muscle cell pairs exhibit bidirectional electrical coupling. Dual innervation of a muscle and nerve cell from a common presynaptic source sometimes occurs and both muscle and nerve cells probably have multiple innervation. Assuming the capability for expression in culture of genetic differences between neurones from the dystrophic and normal chick, we conclude that these differences are not significant in the regulation of synapse formation in dystrophic chick nerve-muscle cell culture.

The discovery of abnormalities of neural function and structure in at least some of the animal and human muscular dystrophies has evoked a fresh evaluation of inherited primary myopathic disease (Harris, 1971). In the mouse with muscular dystrophy (see review by Harman, Tassoni, Curtis, and Hollinshead, 1963) electrophysiological evidence suggests functional disorders of motoneurone, soma, axon, and endplate, as well as of the muscle fibre membrane (Conrad and Glaser, 1964; McComas and Mossawy, 1965; McComas and Mrożek, 1967) and fibre counts of motor nerves are lower in mutant than in control mice (Harris, Wallace, and Wing, 1972). In the chicken with muscular dystrophy (here termed dystrophic chick) there appears to be a decreased release of acetylcholine in the face of normal reserves of that transmitter and slower rates of rise of the muscle action potential (see review by Julian and Asmundson, 1963), both perhaps on the basis of lowered sodium permeability (Albuquerque and Warnick, 1971). In the human, evidence for a neurogenic process

1 Present address: Department of Neurology, Stanford University Medical School, Stanford, California 94305, USA. has been found in Duchenne type muscular dystrophy, dystrophia myotonica, limb-girdle, and facioscapulohumeral muscular dystrophy using a technique of electrical stimulation and recording for the estimation of the number of motor units supplying a muscle (McComas, Campbell, and Sica, 1971; McComas, Sica, and Currie, 1971; Sica and McComas, 1971). In human dystrophia myotonica there is pathological evidence of abnormal motor and sensory innervation of muscle spindles (Daniel and Strich, 1964; Swash, 1972). Much of the impetus for the current conceptual approach to myopathic disease has come from the tremendous surge of interest in neurotrophic regulation of gene expression in muscle (Guth, 1968; Fambrough, 1970; Samaha, Guth, and Albers, 1970; Guth, Samaha, and Albers, 1970; Grampp, Harris, and Thesleff, 1971; Hartzell and Fambrough, 1972; Harris, 1973).

The neurotrophic hypothesis is further supported in the dystrophic mouse by the finding that transplanted muscle exchanged between dystrophic and normal animals degenerates in the case of normal muscle transplanted to a 
dystrophic host and dystrophic muscle reinnervates normally when transplanted to a normal host (Salafsky, 1971); neither is changed when reimplanted beneath the renal capsule instead of a homotopic limb site (Rolston, 1972). Exchanged transplants between dystrophic and normal hamsters similarly degenerate in the dystrophic host but remain unaltered in the normal animal (Jasmin and Bokdawala, 1970), while, in the dystrophic chicken, neither of the exchanged transplants is affected by the new host environments (Cosmos and Butler, 1972). Clearly, there are different regulatory mechanisms in these different animal muscular dystrophies. Vascular (Mendell, Engel, and Derrer, 1971) and immunological (Caspary, Currie, and Field, 1971) factors have also been suggested.

An alternative way of interacting dystrophic and normal nerve and muscle cells in desired proportions is offered by the isolated and controlled environmental system of tissue culture. In addition, new techniques for preparing cultures from single dissociated cells which subsequently grow on the surface of the dish in one or at most a few layers frequently make possible the full pre- and post-junctional visualization of a living nerve and muscle cell under phase contrast microscopy. Using these cultures in conjunction with intracellular microelectrode techniques, Fischbach (1972) has recently demonstrated that chick spinal cord neurones are capable of innervating chick pectoral myotubes in culture and that transmitter release in culture conforms generally to the quantum hypothesis.

In our work we prepared similar cultures, but of dystrophic myotubes and added neurones from either dystrophic or normal embryos. We show a high incidence of synapse formation under both conditions, electrical coupling between nerve and muscle cells, as well as evidence for multiple innervation and discuss these findings with regard to current concepts of muscular dystrophy.

\section{METHODS}

The dystrophic flock of chickens was hatched from eggs given to us by Dr. Louis Pierro, Department of Animal Genetics, University of Connecticut (Storrs, Conn.). All care for the birds and subsequent handling of eggs hatched from them was the responsibility of Truslow Farms in Chestertown, Maryland. The chickens were tested by the State of Maryland for bacterial and viral diseases and none was found, $\bar{z}$ including PPLO. At no time did the chicken feed $\stackrel{\mathbb{D}}{\leftarrow}$ include antibiotics. Both pullets and cockerels showed signs of chicken muscular dystrophy at the time we began using their embryonated eggs for culture. Because the dystrophic mutation which was originally found in the New Hampshire line of $\frac{\hat{S}}{\partial}$ chickens had been backcrossed on the White Leghorn strain at the University of Connecticut, embryos? from normal White Leghorn chickens served as $\Rightarrow$ controls.

Dystrophic and normal 11 day old embryos were used for the dissection of pectoral muscles; 7 day old $\frac{\overline{ }}{\bar{N}}$ embryos were used for the dissection of spinal cords $\frac{\bar{S}}{\vec{Q}}$ and dorsal root ganglia. Dissections were performed $\stackrel{\mathbb{}}{\AA}$ in sterile Puck's saline D1, pH $7 \cdot 2$ and 340 mosmolar, under a stereomicroscope. No more than two $\rightarrow$ embryos either for muscle or spinal cord tissue were dissected before carrying out the remainder of the $\vec{\omega}$ dissociation procedure.

MUSCLE CELL CULTURES The stages in preparation? of muscle cell cultures relatively free of fibroblasts $\omega$ were similar to those described by Fischbach (1972 $\omega$ First single cells were obtained (Shimada, Fischma而, and Moscona, 1969) by a combination of tryps: and mechanical dissociation and filtration (see? 'Dissociation'). The suspension of cells from ofe $\vec{c}$ breast plate (two pectoral muscles) in $18 \mathrm{ml}$. MENS/ 10/10 (see 'Medium') was divided into six tiss culture dishes $(60 \mathrm{~mm}$, Falcon Plastics, no. 3002) a a $\overrightarrow{\mathrm{g}} \overrightarrow{\mathrm{c}}$ incubated at $37^{\circ} \mathrm{C}$ for 30 minutes differentially separate fibroblasts, which rapidly adhere to the plastic, from myoblasts which are still floating in the medium or are only lightly attached after this period of incubation (Yaffe, 1968). The floating myoblasts were decanted and yielded about $6 \times 10^{6}$ viable cells $\stackrel{\circ}{\mathbb{Q}}$ as determined by Nigrosin exclusion. They were $\stackrel{\varrho}{\rightrightarrows}$ plated at $5 \times 10^{5}$ cells $/ 60 \mathrm{~mm}$ dish $\left(2.4 \times 10^{4}\right.$ cells/응 $\mathrm{cm}^{2}$ ) in MEM/10/10. The surface of each dish was coated with $50 \mu \mathrm{g}$ rat tail collagen (Bornstein, 1958) About 48 hours after plating the medium was changed with MEM/2/10 (see 'Medium') containing aminopterin, $4 \times 10^{-7} \mathrm{M}$, in order to reduce proliferation of surviving fibroblasts (Fischbach, 1972; Peacock, 을 Nelson, and Goldstone, 1973). After another 24. hours the aminopterin was replaced with MEM/2/10응 without aminopterin.

MUSCLE PLUS NERVE CELL CULTURes On the fifth $>$ day after the initial plating when there were many․ㅡㄹ. twitching striated myotubes, dissociated cells from N spinal cords and dorsal root ganglia were added at $\mathrm{a}^{\circ}$ density of $2-3 \times 10^{5}$ viable cells $/ 60 \mathrm{~mm}$ dish (approximately $1.2 \times 10^{4}$ cells $/ \mathrm{cm}^{2}$ ). After the addition of 
neurones, some plates received a second incubation in aminopterin medium for 24 hours. Cultures were maintained in a $37^{\circ} \mathrm{C}$ incubator gassed with $5 \%$ $\mathrm{CO}_{2} / 95 \%$ air saturated with water vapour. The medium was changed every two to three days with $3 \mathrm{ml}$. fresh MEM/2/10. Cultures were studied electrophysiologically four to six weeks after the initial plating of myoblasts.

MEDIUM MEM/10/10 consisted of $80 \%$ Eagle's minimal essential medium (MEM) in Earle's salts (Microbiological Associates) plus $10 \%$ chick embryo extract (CEE) and 10\% horse serum (GIBCO). MEM $/ 2 / 10$ was $88 \%$ MEM plus $2 \%$ CEE and $10 \%$ horse serum. Each contained sodium penicillin $\mathrm{G}$, 10 units $/ \mathrm{ml}$., and streptomycin sulphate, $10 \mu \mathrm{g} / \mathrm{ml}$. Both normal and dystrophic 11 day old embryos were used to make respectively normal CEE and dystrophic CEE by incubating a mash of embryonic tissue at a ratio of one embryo $/ 3 \mathrm{ml}$. Earle's salt solution for 24 hours at $4^{\circ} \mathrm{C}$ followed by centrifugation for one hour at 1,200 x $g$ and decantation of the CEE. The embryonic mash was obtained by quickly

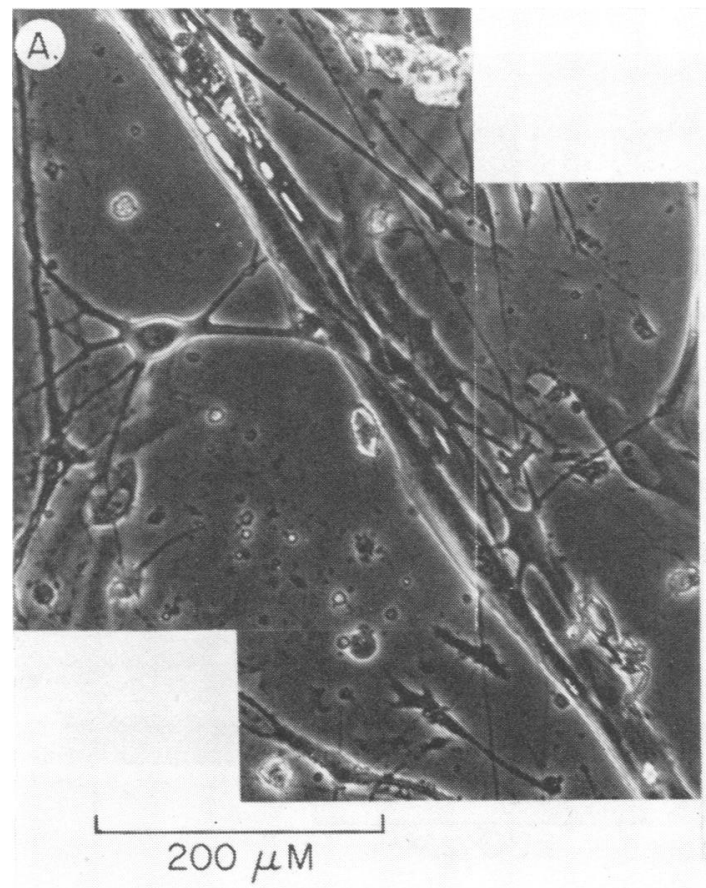

forcing about 12 embryos through the tip of a $30 \mathrm{ml}$. syringe. All medium was freshly made each week and tested bacteriologically before use.

DISSOCIATION Minced fragments of pectoral muscle were dissociated by incubation in Puck's saline D1 containing $0.25 \%$ trypsin (GIBCO) for 30 minutes at $37^{\circ} \mathrm{C}$ followed by removal of the supernatant after centrifugation at $250 \times \mathrm{g}$. The tissue pellet was resuspended in MEM/10/10, triturated with a Pasteur pipette until a milky suspension was obtained, and filtered through a Swinnex filter holder with a double layer of lens paper to remove any tissue fragments. An aliquot of the suspension was counted and plated as outlined above.

Spinal cords and dorsal root ganglia were carried similarly through the above steps to the stage of trituration where first a 20 gauge and then 26 gauge needle was used instead of a pipette. Dissociated neural tissue was not filtered. The yield of cells from one spinal cord and its dorsal root ganglia was about $3 \times 10^{6}$ cells, although it was frequently difficult to

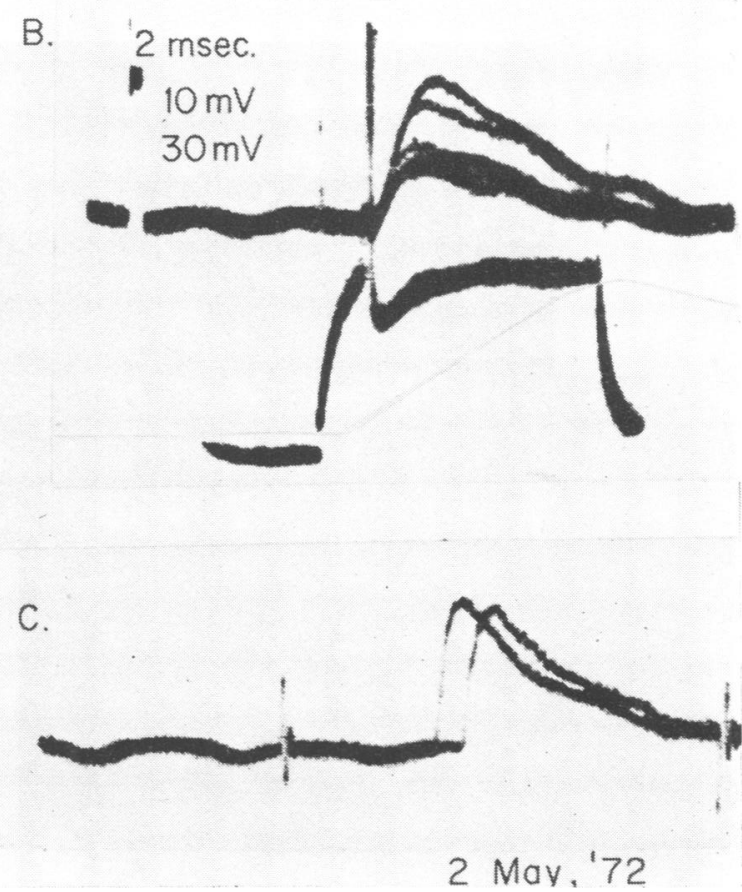

FIG. 1. Evoked PSPs in dystrophic neuromuscular junction. A. Phase contrast photomicrograph of dystrophic neurones and myotubes in a 49 day old culture twice incubated with aminopterin to reduce background cells. B. Successive excitatory PSPs (upper traces) evoked in myotube by action potential firing (lower traces) of neurone. C. PSPs recorded from another myotube innervated by the same neurone as in B. $10 \mathrm{mV}$ calibration pulse in B holds for C. Positive polarity is upgoing in all Figures. 
obtain all the dorsal root ganglia from an embryo and counts varied somewhat.

ELECTROPHYSIOLOGY Details of our techniques for intracellular microelectrode study of cultured cells have been described (Nelson, Peacock, Amano, and Minna, 1969). Briefly, the culture dish is placed on the stage of an inverted phase contrast microscope in

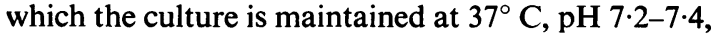
and evaporation is prevented by a thin layer of light mineral oil. Glass capillary pipettes filled with $3 \mathrm{~m} \mathrm{KCl}$ or $4 \mathrm{M} \mathrm{K}$-acetate $(\mathrm{pH} 7)$ are connected in a bridge circuit to an oscilloscope for simultaneous monitoring of transmembrane voltage transients and the current injected across the membrane to produce these transients. In the present experiments an electrode was placed in each of two nearby cells, usually a neurone and a myotube, and the pair was tested for the presence of postsynaptic potentials (PSPs) evoked after action potentials elicited in the neurone by direct electrical stimulation. Of course, spontaneously occurring PSPs could be monitored in myotubes and neurones and on occasion those PSPs evoked by the spontaneous firing of neurones.
Culture medium was always replaced with fresh medium (MEM/2/10) on the day preceding an electrophysiological experiment and cultures were then studied in this medium.

\section{RESULTS}

The data presented here are from dystrophic myotube (DM) cultures to which either neurones from dystrophic embryos, called dystrophic neurones (DN), or from normal embryos, called normal neurones (NN), were added and the combined cultures designated either DN/DM or NN/DM. Normal myotube cultures with added neurones were grown as well but were not studied systematically.

Figure 1A shows a neurone and myotube pair (note microelectrode shadows) in a DN/DM culture relatively free of fibroblasts. Direct electrical stimulation of the neurone elicits action potentials (Fig. 1B, lower trace) each of

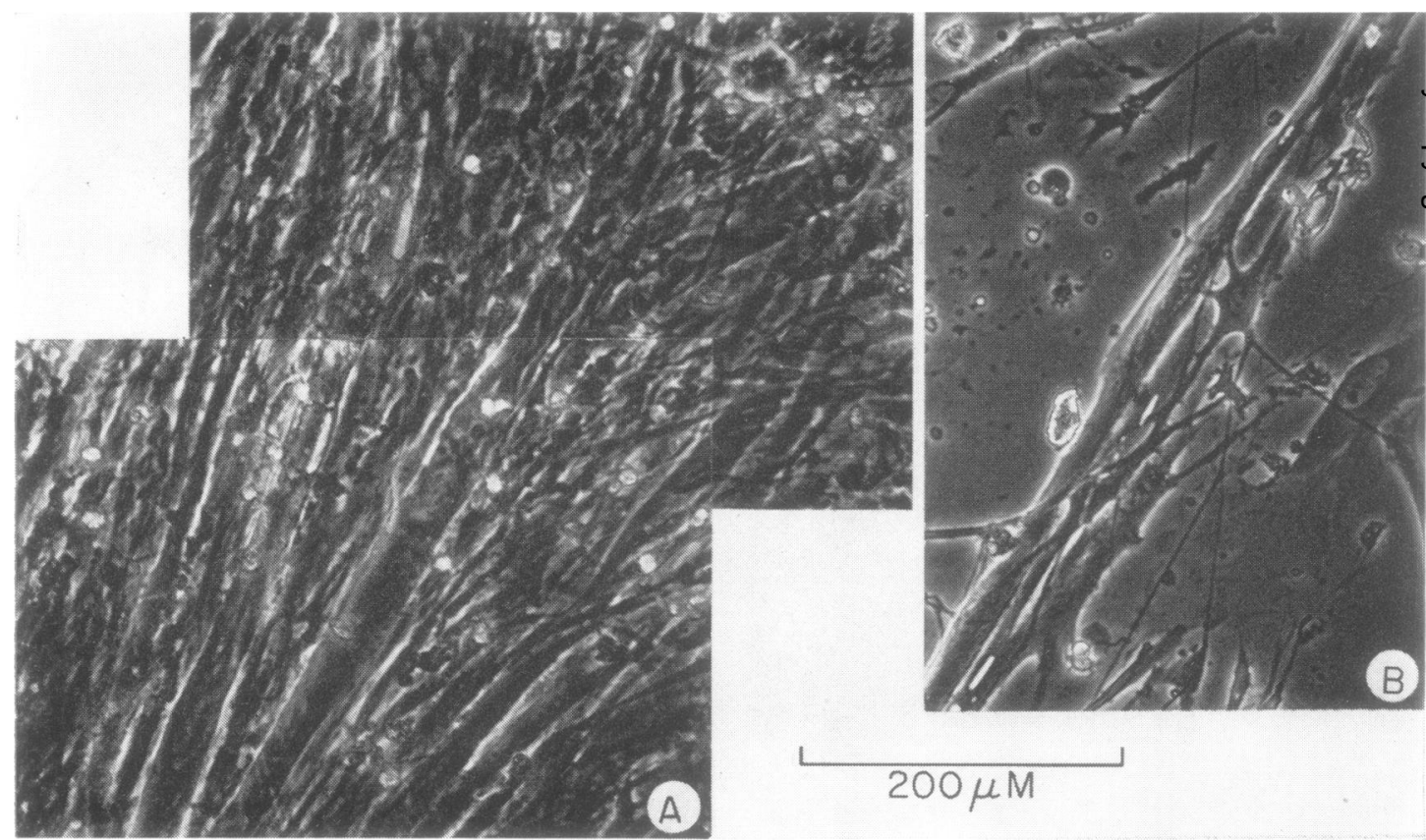

FIG. 2. Single versus double aminopterin incubation. A. Phase contrast photomicrograph of 36 day old dystrophic nerve-muscle cell culture which had a single exposure to aminopterin as a young myoblast culture. B. Phase contrast photomicrograph of 49 day old dystrophic nerve-muscle cell culture which received a second exposure to aminopterin 24 hours after neurones were added to the 5 day old muscle plate. Myotubes here are continuation of field shown in Fig. 1A, but negative reversed in print making. 


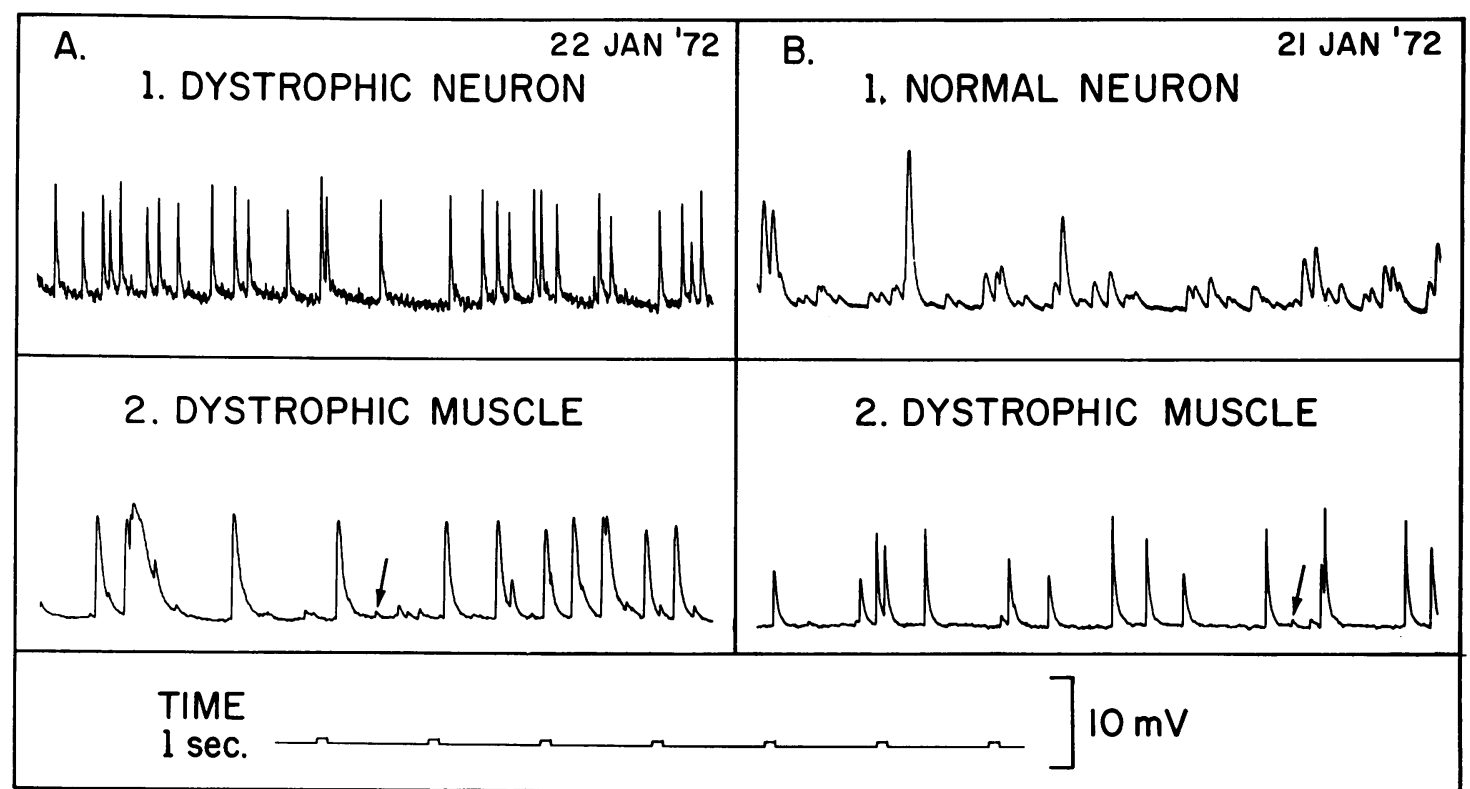

FIG. 3. Spontaneous PSP recordings under various culture conditions. A1 and A2. PSPs recorded at resting membrane potentials of $-58 \mathrm{mV}$ and $-60 \mathrm{mV}$ respectively from a neurone and myotube in a 36 day old $D N / D M$ culture. B1 and B2. PSPs recorded at resting potentials of $-54 \mathrm{mV}$ and $-48 \mathrm{mV}$ respectively from a neurone and myotube in a 35 day old NN/DM culture. PSPs in this Figure were recorded on a penwriter.

which is followed by a PSP recorded from the myotube (Fig. 1B, upper trace). The adjacent, parallel-lying myotube is also innervated (Fig. 1C) by this neurone (note faster rise time of PSP) but not by the neurone overlying the myotubes (Fig. 1A, lower right) suggesting some degree of synaptic specificity. In fact, in these background free cultures, obtained by a second exposure to aminopterin, the incidence of synapse formation $(<5 \%)$ was too low for facile experimentation. Cultures exposed only once to aminopterin had a much higher incidence of synaptogenesis (about $75 \%$ ), even though they had regrown a non-neural background layer after about one month in culture (compare culture in Fig. 2A with that in Fig. 2B). Both cultures were otherwise maintained in low embryo extract medium (Fischbach, 1972) - that is, $\mathrm{MEM} / 2 / 10$.

The spontaneous PSPs recorded from DN/DM cultures are similar to those from NN/DM cultures both for myotubes and neurones (Fig. 3). Neurones most frequently exhibit excitatory PSPs (Fig. 3A1 and B1) but inhibitory potentials are occasionally observed. From some myotubes a low amplitude (about $0.5 \mathrm{mV}$ ) class of PSP (arrows, Fig. 3A2 and B2) was recorded as well as higher amplitude PSPs ( $35 \mathrm{mV}$ maximum). Assuming that the smallest events are miniature endplate potentials, the largest events would have a quantum content of over 70 . The average PSP frequency is about $10 / \mathrm{sec}$ with a range from 2-25/sec.

Similarly, there is little difference as studied in identified synaptic pairs, between PSPs evoked in dystrophic muscle cells by presynaptic stimulation of $\mathrm{DN}$ compared with those evoked by stimulation of NN (Fig. 4). Both DN and NN are fired (Fig. 4A1, second trace, and Fig. 4B1, third trace) by brief current pulses and are followed after a short latency by excitatory PSPs in the myotube. Potentiation of PSP amplitude occurs in each case when a second presynaptic activation follows shortly after the first (Fig. 4A2, lower trace, and Fig. 4B2, third trace).

The incidence of synapse formation (Table 1) is about $75 \%$ for neuromuscular junctions and about $85 \%$ for neural junctions. Resting mem- 


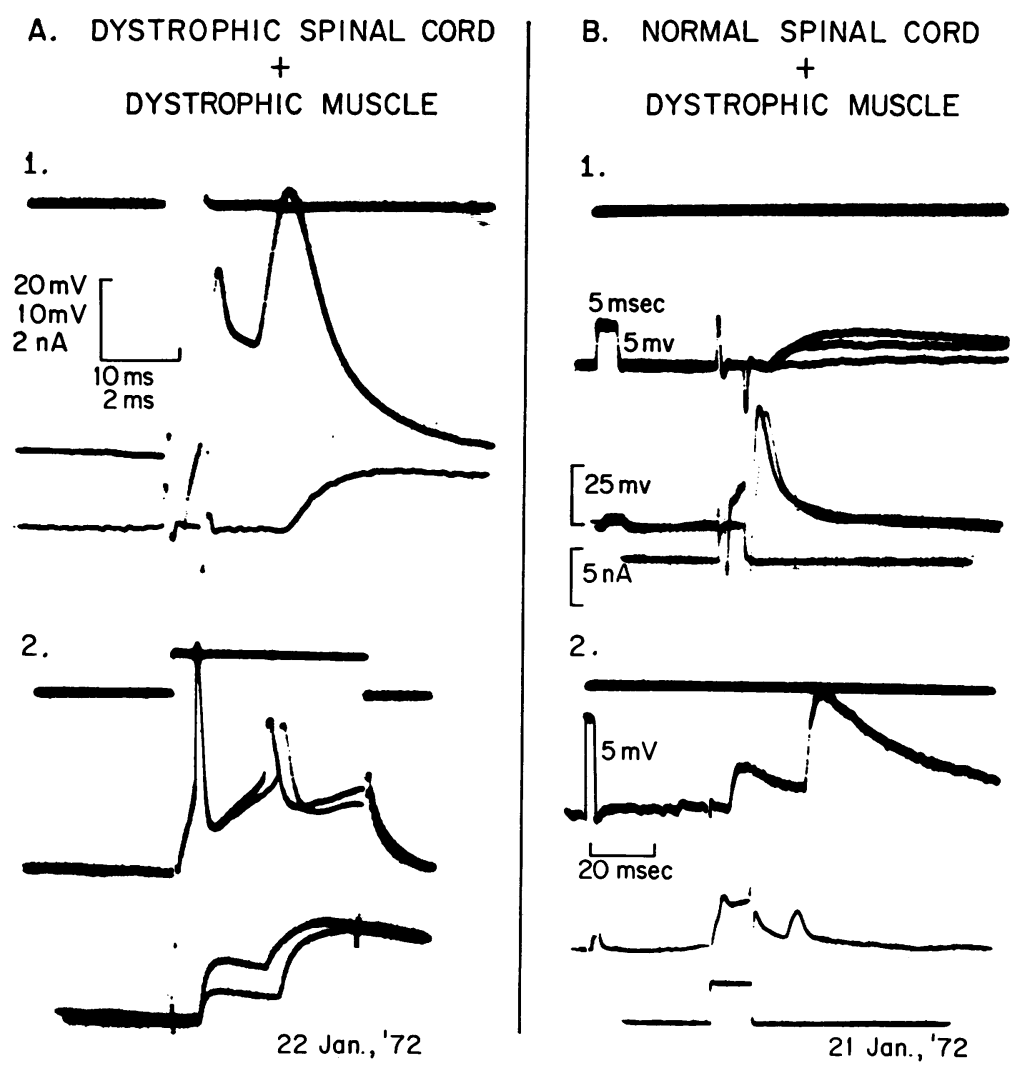

FIG. 4. Evoked PSPs in dystrophic myotubes innervated by dystrophic neurones (A) and normal neurones (B) in cultures respectively 36 and 35 days old. A1. PSP (lower trace) evoked in myotube after action potential firing (middle trace) of neurone stimulated by direct electrical current pulse (upper trace). A2. Same sequence of traces as in A1, but displayed at a slower sweep speed. Membrane potentials of neurone and myotube are $-58 \mathrm{mV}$ and $-50 \mathrm{mV}$ respectively. $20 \mathrm{mV}$ calibration holds for neurone in $\mathrm{A} 1$ and $\mathrm{A} 2$; $10 \mathrm{mV}$ calibration holds for myotube. The time base calibration for $\mathrm{A} 1$ is $2 \mathrm{msec}$ and for A2 it is 10 msec. B1. Successive PSPs (second trace) evoked in myotube by presynaptic firing (third trace) of neurone in response to direct stimulation (bottom trace). Membrane potentials of neurone and myotube are $-80 \mathrm{mV}$ and $-40 \mathrm{mV}$ respectively. B2. Same sequence of traces but PSPs evoked from another myotube by the neurone in $\mathrm{B} 1$. Membrane potential of this myotube is $-58 \mathrm{mV}$.

brane potentials are similar for the classes under comparison so that PSPs are not being obscured or enhanced by differences in the potential driving force. There were no differences which could be ascribed to the use of dystrophic CEE rather than normal CEE in the medium. PSPs frequently trigger action potentials and twitching in myotubes. In these mature cultures spon- taneous contractions without innervation are rare, but, conversely, non-contracting myotubes often are also innervated. Hence twitching per se is not a reliable synaptic marker.

We were surprised to find that about $20 \%$ of nerve-muscle cell pairs (5/25 for DN/DM and 2/11 for NN/DM cultures) exhibited bidirectional electrical coupling (Fig. 5). Coupling can 
A.

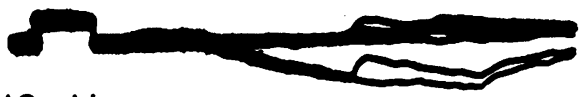

$10 \mathrm{mV}$

$10 \mathrm{msec}$

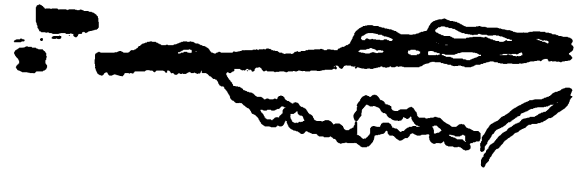

B.

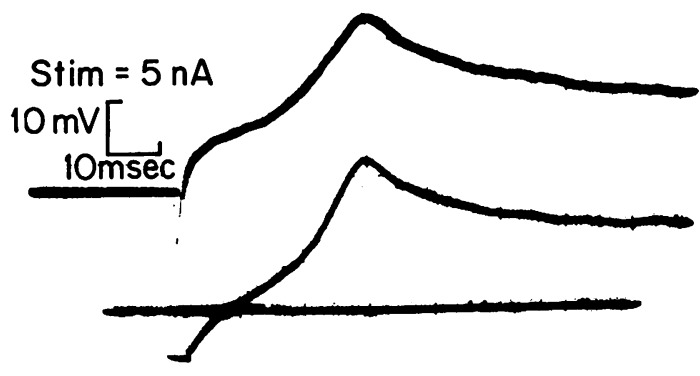

21 Jan., '72

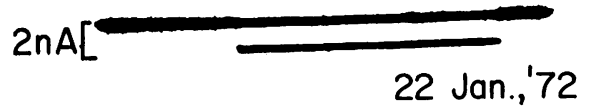

FIG. 5. Electrical coupling between neurones and myotubes. A. Simultaneous hyperpolarizing charging curves elicited in dystrophic neurone (second trace) and dystrophic myotube (third trace) by stimulating current delivered across myotube membrane. Coupling was bi-directional. Membrane potential is - $58 \mathrm{mV}$ for each. Note superimposed spontaneously occurring PSPs. B. Simultaneous local responses recorded from normal neurone (upper trace) and dystrophic myotube (middle trace) to depolarizing current pulses delivered across neuronal membrane. Coupling was again bi-directional. Membrane potentials of neurone and myotube are $-68 \mathrm{mV}$ respectively.

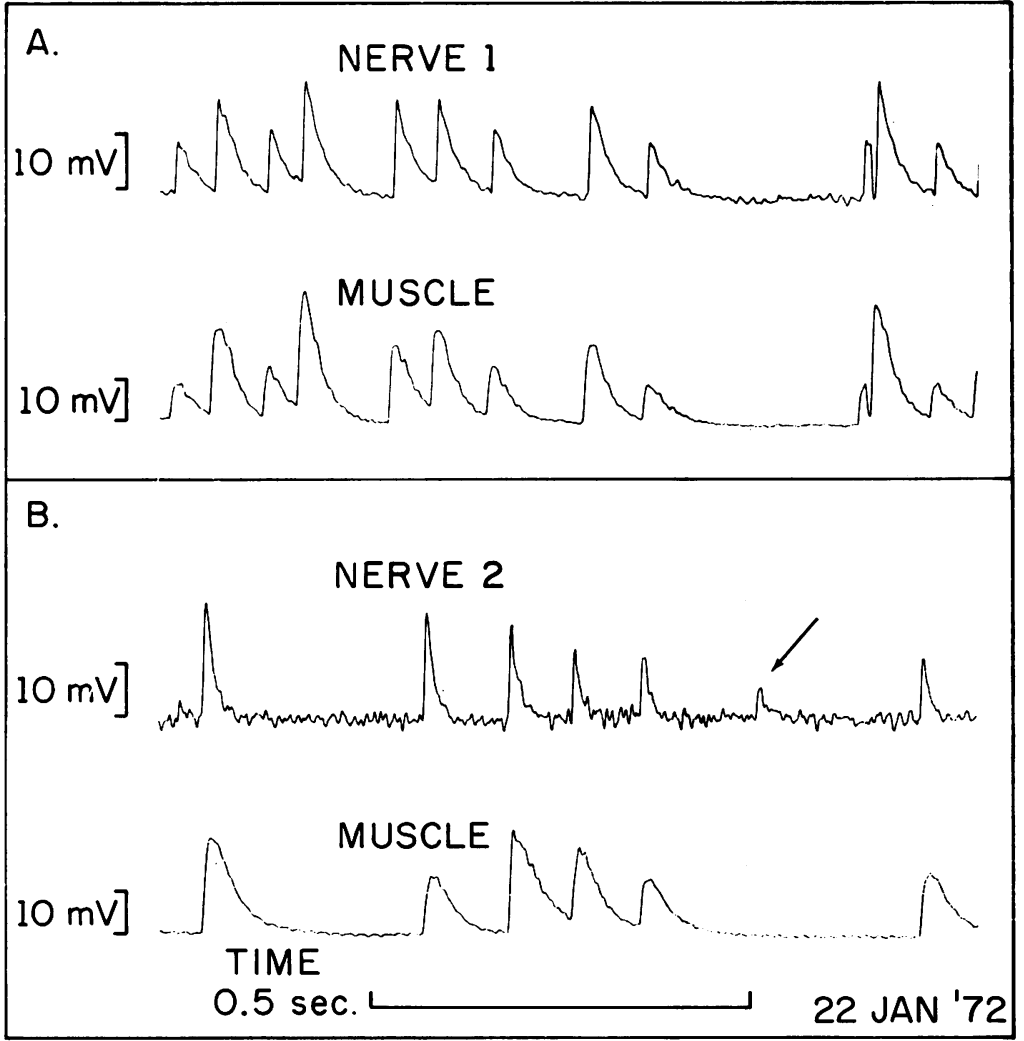

FIG. 6. Spontaneous PSPS recorded from two nerve cells and a myotube in a 36 day old culture. A. Nerve cell 1 and muscle cell are electrically coupled and PSPs are synchronous on each recording. Resting membrane potential of each is $-58 \mathrm{mV}$. B. Nerve cell 2 and same muscle cell as in Fig. $6 \mathrm{~A}$ are not electrically coupled but most PSPs occur synchronously and indicate a common presynaptic input. Additional PSPs (note arrow) are seen on neurone recording. Resting potential of neurone 2 is $-30 \mathrm{mV}$. 
TABLE 1

DYSTROPHIC VERSUS NORMAL INNERVATION OF DYSTROPHIC MYOTUBES

\begin{tabular}{ccccc}
\hline \multicolumn{2}{c}{ Cell type } & $\begin{array}{c}\text { PSPs* } \\
(\%)\end{array}$ & $\begin{array}{c}\text { Postsynaptict } \\
\text { RMP }(-m V)\end{array}$ & $n$ \\
\cline { 1 - 4 } Presynaptic & Postsynaptic & & & \\
\hline D-nerve & D-muscle & 77 & 52 & 43 \\
N-nerve & D-muscle & 75 & 53 & 27 \\
D-nerve & D-nerve & 87 & 43 & 30 \\
N-nerve & N-nerve & 82 & 43 & 11 \\
\hline
\end{tabular}

* Average PSP frequency 8-10/sec (range 2-25/sec).

$+\mathrm{SD} \pm 10.5$ for each category.

be demonstrated with either de- or hyperpolarizing current stimulation of either nerve or muscle cell and recording simultaneously occurring changes in membrane potential in both members of the pair. Both soma and process coupling to myotubes occur. In most instances the coupling ratio is nearly unity and rectification between nerve and muscle or vice versa is minimal.

Complex patterns of synaptic interaction were seen, as in Fig. 6 for example, which required a network of at least three neurones and one myotube to explain the data. There is electrical coupling between the nerve-muscle cell pair in Fig. 6A and common innervation of the nervemuscle cell pair in Fig. 6B (note that this is the same muscle cell as in Fig. 6A but the muscle cell is not electrically coupled to nerve 2). We therefore conclude that a third neurone innervated both nerve cell 2 and the myotube which in turn was electrically coupled to nerve cell 1 . A fourth neurone may be involved which produced the PSP (arrow in Fig. 6B) which was recorded from nerve 2 but not from the muscle. Alternatively this PSP may represent a spontaneous, miniature PSP but it is rather large to be explained on this basis.

Common innervation of simultaneously recorded cell pairs is a prominent feature of the cultures and data regarding this point are summarized in Table 2 (note that these were cell pairs which were not electrically coupled). It is evident that both dystrophic and normal neurones are capable of simultaneous synapse formation with at least one muscle and neuronal cell type or with pairs of muscle cells. In four of 12
TABLE 2

COMMON INNERVATION OF NERVE-MUSCLE CELL PAIRS (CELL PAIRS NOT COUPLED ELECTRICALLY)

\begin{tabular}{llc}
\hline Culture condition & Cell pairs $(n)$ & $\begin{array}{c}\text { Pairs with } \\
\text { synchronous PSPs } \\
(\%)\end{array}$ \\
\hline D-nerve/D-muscle & $\begin{array}{l}\text { Nerve-muscle (20) } \\
\text { Muscle-muscle (5) }\end{array}$ & $\begin{array}{c}7(35) \\
3(60)\end{array}$ \\
N-nerve/D-muscle & $\begin{array}{l}\text { Nerve-muscle (9) } \\
\text { Muscle-muscle (7) }\end{array}$ & $\begin{array}{c}1(11) \\
\end{array}$ \\
\hline
\end{tabular}

simultaneously recorded pairs of muscle cells, in addition to the synchronously occurring PSPs, one cell of the pair exhibited PSPs which were not accompanied by PSPs in the other cell of the pair. Either the muscle with the 'extra' PSPs was multiply innervated, or occasional failure of transmission occurred at the junction between the neurone innervating the two fibres and the other muscle fibre.

\section{DISCUSSION}

A major finding of the present work is the hig还 incidence of neuromuscular synapse formatio in dissociated cultures of dystrophic muscle cells to which neurones from dystrophic and normal chicks have separately been added. The dystrophic muscle cultures were prepared from the pectoral muscle, the earliest and most severely affected muscle in chicken muscular dystrophy which genetically has an autosomal recessive pattern of inheritance with complete penetrance. Hence on a genetic basis this embryonic muscle tissue has a myopathic destiny if it remains in situ. We failed to find an electrophysiological difference in synaptogenesis under the two culture conditions with regard to incidence (about $75 \%$ ), spontaneous PSP frequency (about 10/ $\mathrm{sec}$ ), and maximum quantum content (about 70 ). These values compare respectively with $5-10 \%$ innervation incidence, PSP frequency $1-10 / \mathrm{min}$., and maximum quantum content (about 30) reported by Fischbach (1972) for nerve-muscle junctions in cell cultures from normal White Leghorn chick embryos. The major difference in the preparation of our cultures from the cultures 
in the Fischbach study was the inclusion of dorsal root ganglion cells in the neuronal population. Our cultures were over a month old at the time of the electrophysiological experiments. Non-neuronal cells were again present, perhaps also glial or Schwann cells; fibroblasts were present in these cultures as well, presumably. Many of these cells could be eliminated by a second exposure to aminopterin but with a drastic fall in the incidence of synapse formation. Another attractive hypothesis is that the second aminopterin incubation interrupts a vulnerable period of synaptic specification of the nascent neurite-myotube contacts.

Dystrophic muscle cell cultures which are not treated with mitotic inhibitors have been reported to grow faster than normal in the first week in culture, then progressively larger numbers of myotubes show degenerative changes and in the third week myotubes have largely disappeared from the cultures (Askanas, Shafiq, and Milhorat, 1971). We have not repeated that experiment, but have had no difficulty in maintaining the aminopterin treated cultures for at least a month. Perhaps here again the drug interrupts a vulnerable metabolic period, in this case for myotube growth.

The innervation of both myotube and neurone from a common source (Fig. 6) probably is indicative of the existence of cholinoceptive neurones in these cultures. We did not use acetylcholine blocking agents, but d-tubocurarine blocks synaptic potentials at the neuromuscular junction in culture (Fischbach, 1972) and, according to the single transmitter principle of Dale, the presynaptic endings on this set of neurones sharing inputs with muscle cells, should also be releasing acetylcholine. Since the commonly known class of cholinoceptive neurones in spinal cord, the Renshaw cells, would probably be too small for satisfactory intracellular recordings, our findings are indicative of another class of cholinoceptive spinal neurones.

Two other features of the cultures in our series, electrical coupling between nerve-muscle pairs and multiple innervation of muscle cells have been found in low incidence in normal nerve-muscle cell cultures (Fischbach, 1972) and the suggestion has been made that these findings represent early, possibly embryonic, stages of synapse formation. In our well-established and morphologically mature cultures, it is possible that the electrical coupling and multiple innervation represents a persistence of early stages of synaptogenesis. Persistence of embryonic properties has been reported for dystrophic chick muscle by several investigators (see Wilson, Kaplan, Merhoff, and Mori, 1970). This lack of repression of the embryonic state in mature cultures could well be specified by the dystrophic myotube. Certainly the bi-directional electrical coupling indicates that a mechanism exists for the exchange of molecular information between nerve and muscle cells. Our data lead us to speculate that the dystrophic myotube is regulating the expression of neuronal characteristics and that a myotypic rather than a neurotrophic phenomenon is responsible for our results.

\section{REFERENCES}

Albuquerque, E. X., and Warnick, J. E. (1971). Electrophysiological observations in normal and dystrophic chicken muscles. Science, 172, 1260-1263.

Askanas, V., Shafiq, S. A., and Milhorat, A. T. (1971). Normal and dystrophic chicken muscle at successive stages in tissue culture. Archives of Neurology, 24, 259-265.

Bornstein, M. B. (1958). Reconstituted rat-tail collagen used as a substrate for tissue culture on coverslips on Maximov slides and roller tubes. Laboratory Investigation, 7, 134-137.

Caspary, E. A., Currie, S., and Field, E. J. (1971). Sensitized lymphocytes in muscular dystrophy: evidence for a neural factor in pathogenesis. Journal of Neurology, Neurosurgery, and Psychiatry, 34, 353-356.

Cosmos, E., and Butler, J. (1972). Differentiation of muscle transplanted between normal and dystrophic chicks. In Research in Muscle Development and the Muscle Spindle, pp. 149-162. Edited by B. Q. Banker, R. L. Przybylski, J. P. Van Der Meulen, and M. Victor. International Congress Series No. 240. Excerpta Medica: Amsterdam.

Daniel, P. M., and Strich, S. J. (1964). Abnormalities in the muscle spindles in dystrophia myotonica. Neurology (Minneap.), 14, 310-316.

Fambrough, D. M. (1970). Acetylcholine sensitivity of muscle fiber membranes: mechanism of regulation by motoneurons. Science, 168, 372-373.

Fischbach, G. D. (1972). Synapse formation between dissociated nerve and muscle cells in low density cell cultures. Developmental Biology, 28, 407-429.

Grampp, W., Harris, J. B., and Thesleff, S. (1971). Inhibition of denervation changes in mammalian skeletal muscle by actinomycin D. Journal of Physiology, 217, 47P-48P.

Guth, L. (1968). 'Trophic' influence of nerve on muscle. Physiological Reviews, 48, 645-687.

Guth, L., Samaha, F. J., and Albers, R. W. (1970). The neural regulation of some phenotypic differences between the fiber types of mammalian skeletal muscle. Experimental Neurology, 26, 126-135.

Harman, P. J., Tassoni, J. P., Curtis, R. L., and Hollinshead, M. B. (1963). Muscular dystrophy in the mouse. In Muscular Dystrophy in Man and Animals, pp. 407-456. Edited by G. H. Bourne and M. N. Golarz. Karger: Basel. 
Harris, A. J. (1973). Role of acetylcholine receptors in synapse formation. Journal of General Physiology. (In press.)

Harris, J. B. (1971). The relation of trophic influences to diseases of muscle. Developmental Medicine and Child Neurology, 13, 669-671.

Harris, J. B., Wallace, C., and Wing, J. (1972). Myelinated nerve fibre counts in the nerves of normal and dystrophic mouse muscle. Journal of Neurological Sciences, 15, 245249.

Hartzell, H. C., and Fambrough, D. M. (1972). Acetylcholine receptors. Distribution and extrajunctional density in rat diaphragm after denervation correlated with acetylcholine sensitivity. Journal of General Physiology, 60, 248262.

Jasmin, G., and Bokdawala, F. (1970). Muscle transplantation in normal and dystrophic hamsters. Revue Canadienne de Biologie, 29, 197-201.

Julian, L. M., and Asmundson, V. S. (1963). Muscular dystrophy of the chicken. In Muscular Dystrophy in Man and Animals, pp. 457-498. Edited by G. H. Bourne and M. N. Golarz. Karger: Basel.

McComas, A. J., and Mossawy, S. J. (1965). Electrophysiological investigation of normal and dystrophic muscles in mice. In Research in Muscular Dystrophy. The Proceedings of the Third Symposium of the Muscular Dystrophy Group, London, 1965. Pp. 317-341. Pitman Medical: London.

McComas, A. J., and Mrożek, M. (1967). Denervated muscle fibres in hereditary mouse dystrophy. Journal of Neurology, Neurosurgery, and Psychiatry, 30, 526-530.

McComas, A. J., Sica, R. E. P., and Currie, S. (1971). An electrophysiological study of Duchenne dystrophy. Journal of Neurology, Neurosurgery, and Psychiatry, 34, 461-468.

McComas, A. J., Campbell, M. J., and Sica, R. E. P. (1971). Electrophysiological study of dystrophia myotonica. Journal of Neurology, Neurosurgery, and Psychiatry, 34, 132-139.

Mendell, J. R., Engel, W. K., and Derrer, E. C. (1971).
Duchenne muscular dystrophy: functional ischemia reproduces its characteristic lesions. Science, 172, 1143-Z 1145.

Nelson, P. G., Peacock, J. H., Amano, T., and Minna, J. (1971). Electrogenesis in mouse neuroblastoma cells in vitro. Journal of Cellular Physiology, 77, 337-352.

Peacock, J. H., Nelson, P. G., and Goldstone, M. W. (1973). Electrophysiologic study of cultured neurons dissociateden from spinal cords and dorsal root ganglia of fetal mice. Developmental Biology, 30, 137-152.

Rolston, J. L. L. (1972). Muscle transplantation into mice with muscular dystrophy. Archives of Neurology, 26, 258-.. 264.

Salafsky, B. (1971). Functional studies of regenerated muscles $\overrightarrow{\bar{\sim}}$ from normal and dystrophic mice. Nature, 229, 270-272.

Samaha, F. J., Guth, L., and Albers, R. W. (1970). The neural regulation of gene expression in the muscle cell. Experimental Neurology, 27, 276-282.

Shimada, Y., Fischman, D. A., and Moscona, A. A. (1969). Formation of neuromuscular junctions in embryonic cell cultures. Proceedings of the National Academy of Sciences 0 of the United States of America, 62, 715-721.

Sica, R. E. P., and McComas, A. J. (1971). An electrophysiological investigation of limb-girdle and facioscapulo- $\overrightarrow{\vec{\omega}}$ humeral dystrophy. Journal of Neurology, Neurosurgery, and Psychiatry, 34, 469-474.

Swash, M. (1972). The morphology and innervation of the muscle spindle in dystrophia myotonica. Brain, 95, 357-? 368.

Wilson, B. W., Kaplan, M. A., Merhoff, W. C., and Morg S. S. (1970). Innervation and regulation of acetylcholi esterase activity during the development of normal afd dystrophic chick muscle. Journal of Experimental Zoologe्f, 174, 39-54.

Yaffe, D. (1968). Retention of differentiation potentialiti底 during prolonged cultivation of myogenic cells. Proceeg- $c$ ings of the National Academy of Sciences of the Unitegd States of America, 61, 477-483. 